\title{
Applications of microscopic techniques in life sciences: selected contributions of the Microscopy Conference 2009 in Graz
}

\author{
Ursula Lütz-Meindl
}

Received: 30 July 2010 / Accepted: 30 July 2010

(C) Springer-Verlag 2010

This special issue of Protoplasma includes a selection of original papers presented during the "Life Science" section of the Microscopy Conference 2009 in Graz from August 30 until September 4. For the first time, this congress was organized as joint meeting of the "Dreiländertagung" and the "Multinational Congress on Microscopy" by scientists of the University of Graz headed by Ferdinand Hofer and the conference president Peter Karnthaler under the participation of the following societies: Austrian Society for Electron Microscopy, Croatien Microscopy Society, Czechoslovak Microscopy Society, German Society for Electron Microscopy, Hungarian Society for Microscopy, Italian Society of Microscopical Sciences, Serbian Society for Microscopy, Slovene Society for Microscopy, and Swiss Society for Optics and Microscopy.

The organizers successfully assembled a well-balanced scientific program, including the three main sections "Instrumentation and Methodology", "Life Sciences", and "Material Sciences", which was presented in more than 650 contributions and attracted the interest of more than 1,000 participants.

The "Life Science" section covered a broad range of microscopic applications starting from the tracking of a single molecule to the $3 \mathrm{D}$ depiction of cells and tissues in the fields of microbiology, plant and animal biology as well as medicine. The section was subdivided into the symposia "3D and cryo-transmission electron microscopy", "High resolution light microscopy, correlative light and electron microscopy", "Tracking molecules in vivo, intracellular

U. Lütz-Meindl $(\square)$

Cell Biology Department, University of Salzburg,

Hellbrunnerstrasse 34,

Salzburg 5020, Austria

e-mail: Ursula.LUeTZ-MEINDL@sbg.ac.at trafficking and cellular dynamics", "Microscopy in plant sciences and microbiology", "Microscopy in developmental biology and medicine", "Structure of cells and tissues, localization of molecular targets", and "Image processing for life sciences."

The selected peer-reviewed papers of the present issue highlighted new findings in life sciences obtained by application of different microscopic techniques. In the first methodological part, a new computer software tool is employed by Kreft et al. to analyze intensity profiles of fluorescent probes in confocal images by using rat skeletal muscle fibers as examples. The publication by Leser et al. demonstrates the capability of a focused ion beam/scanning electron microscopy system for overall examination of epithelial tissues of an isopod under normal, stress, or pathological conditions. A non-invasive method for imaging of biominerals and cell wall anisotropy changes using a LCPolScope is introduced by Eder et al. and demonstrates this method as an attractive tool for studying dynamic ordering of biomolecules under certain conditions. The following article by Rancic et al. leads over to the plant part by characterizing the hydraulic network of tomato fruit pedicels by application of various techniques including light and fluorescence microscopy, transmission (TEM) and scanning electron microscopy (SEM), maceration, tissue clearing, and X-ray computed tomography. A selection of different plant surfaces is shown in the article of Stabentheiner et al. demonstrating the potential of environmental SEM for investigating hydrated and uncoated plant samples. A short communication by Sassmann et al. compares copper resistance of two different moss species by analyzing their detailed growth rates. Subcellular localization of glutathione as an important antioxidant and redox buffer in roots and leaves of different higher plants and in cyanobacteria by immuno-TEM is the subject of the two studies by Zechmann et al. and Zechmann 
and Müller. In the animal cell biological and medical parts of this issue, morphological criteria are set, and cell adhesion proteins are immuno-localized by TEM in the article by Lokar et al. to distinguish membrane nanotubes from filopodia, and a model of nanotube attachment is proposed. The review by E. Kreft et al. summarizes recent discoveries on the role of tight junctions, dynamics of the Golgi apparatus and post-Golgi compartments, and intracellular trafficking during the biogenesis and maintenance of the blood-urine barrier. In an ultrastructural study by Matysiak and Jodlowska-Jedrych, morphological changes in the adrenal cortex are investigated as a consequence of the administration of a non-steroidal anti-inflammatory drug. Furthermore, the article by Jodlowska-Jedrych et al. shows that high doses of medroxyprogesterone have inhibiting effects on the synthesis of binding proteins and cause a loss of the oocyte contact with the external glycoprotein membrane, the zona pellucida. Finally, the contribution by Bizak Mali and Bulog gives insight into ultrastructural changes during oogenesis in the neotenic cave dwelling salamander Proteus anguinus anguinus.

As the guest editor of this special issue, I would like to thank the editor in chief of Protoplasma, Peter Nick, for the opportunity to realize this publication as well as Petra Hohenberger from the editorial office and Amrei Strehl from Springer-Verlag for numerous helpful advices and reminders during and prior to the preparation of this volume. I am grateful to all authors for their valuable contributions and constructive cooperation and to all reviewers for their time and both their critical as well as their encouraging comments which improved the manuscripts. 